\title{
Lung cancer Prediction and Classification based on Correlation Selection method Using Machine Learning Techniques
}

\author{
$1^{\text {st }}$ Dakhaz Mustafa Abdullah \\ Technical College of Informatics, Akre \\ Duhok Polytechnic University \\ Duhok, Iraq \\ dakhaz.abdullah@dpu.edu.krd
}

\author{
$2^{\text {nd }}$ Adnan Mohsin Abdulazeez \\ Presidency of Duhok Polytechnic \\ University \\ Duhok Polytechnic University \\ Duhok, Iraq \\ adnan.mohsin@dpu.edu.krd
}

\author{
$3^{\text {rd }}$ Amira Bibo Sallow \\ College of Engineering \\ Nawroz University \\ Duhok, Iraq \\ amira.bibo@nawroz.edu.krd
}

https://doi.org/10.48161/qaj.v1n2a58

\begin{abstract}
Lung cancer is one of the leading causes of mortality in every country, affecting both men and women. Lung cancer has a low prognosis, resulting in a high death rate. The computing sector is fully automating it, and the medical industry is also automating itself with the aid of image recognition and data analytics. This paper endeavors to inspect accuracy ratio of three classifiers which is Support Vector Machine (SVM), KNearest Neighbor (KNN)and, Convolutional Neural Network (CNN) that classify lung cancer in early stage so that many lives can be saving. Basically, the informational indexes utilized as a part of this examination are taken from UCI datasets for patients affected by lung cancer. The principle point of this paper is to the execution investigation of the classification algorithms accuracy by WEKA Tool. The experimental results show that SVM gives the best result with $95.56 \%$, then CNN with CNN $92.11 \%$ and KNN with $88.40 \%$.
\end{abstract} CNN.

Keywords - Lung Cancer, Machine Learning, SVM, KNN,

\section{INTRODUCTION}

Cancers exist in several organs, and simultaneously, and different types of cancer occur in various organs of the body. The illness may even go unnoticed for long periods of time. According to WHO reports, cancer may be prevented if it is detected early enough. The patient's life span will be extended whether he or she receives an early prognosis [1][2][3][4]. Lung cancer has a low prognosis that differs greatly depending on tumor staging at the time of diagnosis. Lung cancer is divided into two types of clinical practice: non-small cell lung cancer (NSCLC) and small cell lung cancer (SCLC) [5][6]. It is, in reality, a malignant tumor characterized by unregulated cell tissue formation. Lung cancer developed mostly as a result of long-term tobacco use [7]. According to research, a stable individual may be affected by nineteen distinct forms of cancer. Lung cancer has the largest death rate among all of these tumors. This disease is expected to kill over 1.7 million people per year [8]. In the area of machine learning (ML) research has already grown a great deal, which is helpful to reduce human laborers. ML combines statistics and computers in the area of artificial intelligence to create algorithms that become more efficiently when subject to relevant data[9][10]. Many systems lack adequate detection accuracy, and some systems must also be developed in order to reach the highest accuracy of $100 \%$. Pulmonary cancer identification and classification were based on machine learning techniques and image processing techniques [11]. However, some signs of lung cancer patients, such as their smoking rate, may aid in early detection of the disease [12][13][14]. Researchers started to use machine learning for medical diagnosis after the advent of artificial intelligence. using a machine learning approach to investigate the classification of diseases in traditional Chinese medicine clinical data (TCM). Valuable guidelines on diagnosis of brain disturbances from network architecture aspects, function learning and classification prediction via the method of machine learning, and provided through the machine learning method and the implementation of the brain network based on machine learning [15]. It will be a key step towards improved early detection [16].

This paper provides an effective method to predict lung cancer in early stage with heigh accuracy ratio. The dataset used is taken form UCI machine learning repository. Then apply three classifier Support Vector Machine (SVM), KNearest Neighbor (KNN)and, Convolutional Neural Network $(\mathrm{CNN})$ to endeavors inspect accuracy ratio of three classifier by using WEKA tool. the present study is aid to develop a Machine Learning Models to detect the lung cancer with better accuracy.

This paper is organized as follows. Section 2 introduce to lung cancer. Section 3 Material \& Methods that used in this paper. describes related work in Section 4. then Section 5 present the theory, introduction to machine learning and their types also confusion matrix. Section 6 present the Performance evaluation and results. Finally, Section 7 Conclusion.

\section{LUNG CANCER}

Carcinogenesis is the unchecked proliferation of one or more cell types. Good tissues do not support the growth of 
normal cells, and when they do, they separate quickly and become tumors. Primary lung cancer originates elsewhere in the body and spreads to the lungs, while secondary lung cancer starts elsewhere in the body and then spreads from there. It's one of the most aggressive types of cancer and a life-threatening threat to the human body [17]. If this unchecked development can be identified correctly at an early point, it can help to diagnose the likelihood of unnecessary surgery and improve the chance of recovery. Chronic Obstructive Pulmonary (COPD) illness attacks the areas of the lungs and causes diseases such as measles, influenza, pneumonia, and other respiratory issues such as asthma. Small Cell Lung Cancer (SCLC) or oat cell cancer and Non-Small Cell Lung Cancer (NSCLC) are the two main forms of lung cancer that develop and expand in separate ways and may be handled accordingly. Within the non-small cell lung cancer category, there are three subtypes (adenocarcinomas, squamous cell carcinomas, large cell carcinomas) fig (1) show the two types of lung cancer. So Mixed small cell/large cell cancer is a disease that occurs where a patient shows symptoms of both types of cancer. (NSCLC) Adenocarcinoma is more common and progresses more slowly than small cell lung cancer. Small cell lung cancer is linked to smoking which progresses more rapidly by becoming a large tumor that will spread across the body [18][19].

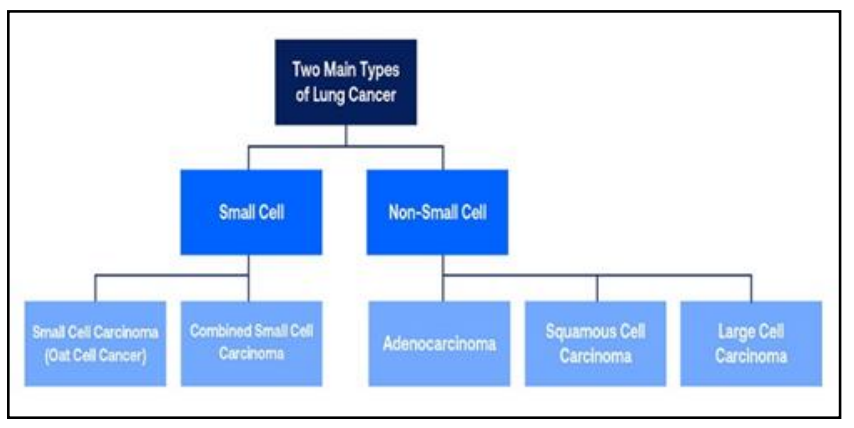

Fig. 1. Lung Cancer Tupes

\section{MACHINE LEARNING}

Machine learning is a subfield of Artificial Intelligence [20]. Machine Learning is also used for complex data classification and decision making [21][22]. In general, the implementation of algorithms aids the machine's learning. Machine learning gives systems the opportunity to learn automatically and improve over time without being directly configured. The implementation of algorithms aids the computer in learning and making the required decisions [11][23]. Machine Learning strategies and activities are narrowly divided into three categories:

\section{a. Supervised learning}

Machine learning, in its most simple form, employs programmed algorithms that learn and refine their functions by processing input data and making predictions within a reasonable range. These algorithms aim to be predictive more precisely by feeding fresh data[24][25]. While there are several changes in the way machine learning algorithms are grouped. Two categories of issues: grouping problems and back-up problems, are well suited to supervised learning algorithms. The output variable usually takes on a limited number of discrete values[26][27].

\section{b. Un-Supervised Learning}

Unsupervised Learning is a form of learning that occurs without the presence of a supervisor [28]. The machine is given some sample inputs, but no output is generated in the method of learning. Since there is no optimal value over here, categorization is used to ensure that the algorithm distinguishes between the datasets correctly. It is the difficulty of finding unknown structure in unidentified details [29][30]. Although there are no testing sets or tests given to the respondent, there are no opportunities to reward a successful solution. Unlike supervised learning and reinforcement learning, unsupervised learning has no teacher, and produces results that are unrelated to prior experience. It is directly connected to density and statistics [21][31].

\section{c. Reinforcement Learning}

Reinforcement Learning, this machine learning style comes from interacting with its surroundings Reinforcement learning. A Reinforcement Learning manager learns from the meaning of tasks, and even by explicitly articulated instructions, and decides on previous behaviors by using new techniques. Since specific input/output data sets are not provided, this differs from traditional supervised learning. Instead, the focus is on the presentation, which entails striking a balance between discovery (of uncharted territory) and utilization (of existing data) [32][33].

\section{DEEP LEARNING}

Deep learning is a type of machine learning techniques that uses representation learning to categorize important features for classification problems [6]. The primary characteristic of deep learning is its compatibility with features, although it may also learn from data. So, to learn complex features a deep learning integrates the simple features that have learned from data. Deep learning is accomplished using multiple-layer artificial neural networks, such as the Deep Neural Network (DNN), Convolutional Neural Network (CNN), and the Recurrent Neural Network (RNN) [13][23].

\section{RELATED WORKS}

Roy et al[34]. They use a combination of image processing biomedical techniques and information discovery in data to improve accuracy and assess precise significance for early detection of lung carcinoma. The representation of the lungs acquired from CT (Computer Tomography) The scan images are pre-processed, and the Region of Interest is segmented (ROI) is performed. The Random Forest procedure is used to distinguish the distinct features. Using an SVM Classifier, the SURF (Speeded Up Robust Functionality) algorithm was used to extract features like entropy, co-relation, power, and variance from Saliency Enhanced images. The image's classification determines if it is safe or toxic (carcinomic). CT scan images were used as the dataset. The SVM classification and random forest algorithm were used to carry out the whole operation. Using SVM classification, the best outcome is achieved. This technique is 94.5 percent effective in general, 74.2 percent sensitive, 66.3 percent recall, and $77.6 \%$ specific.

For lung cancer diagnosis, Faisal et al [12] recommend evaluating machine learning classifiers as well as, classifiers such as Multilayer perceptron (MLP), Nave Bayes, Decision 
Tree, Neural Network, Gradient Boosted Tree, and SVM are evaluated. The dataset was downloaded from the UCI registry and is used to analyze random forest and plurality voting-based ensembles for predict lung cancer. Gradient Boosted Tree was found to outperform all other person and ensemble classifiers. Gradient-boosted Tree outperformed all others as well as ensemble classifiers, achieving 90\% precision, according to performance assessments.

Delta Radiomics uses the machine learning methods proposed by Baskar et al [35] to extract the characteristics of the cancer nodules. Lung cancer nodule malignancy is predicted by using the Support Vector Machine (SVM). The SVM can examine compact features in a lung cancer nodule photograph, and image classification is useful in distinguishing between the multiple nodules. As a result, SVM is recommended as the best tool for diagnosing and detecting lung cancer, with a 90.9 percent accuracy rate.

Boban et al [36]. They use ML algorithms for the 400 lung disease videos, including the Multilayer perceptron (MLP), KNN and SVM classifiers (i.e., CT scan images). The performance is segmented after extraction of features and compares the exactness of the classifier. When a classifier has received a CT scan image, it contains irrelevant content. Gray Level Cooccurrence Matrix (GLCM) is used to pick the most important features (i.e., for removing features). This classification is $98 \%$ accuracy for MLP, 70,45\% for SVM accuracy, and 99,2\% for KNN accuracy.

Using Deep Learning, Sreekumar et al [37] proposed a method for detecting malignant pulmonary nodules from CT scans. To block out the lung areas from the scans, a preprocessing pipeline was used. A 3D CNN model based on the $\mathrm{C} 3 \mathrm{D}$ network architecture was used to remove the functionality. For the decrease of false-positives, researchers used the Lung Image Database Consortium (LIDC-IDRI) as well as a few materials from the LUNA16 grand challenge. The end result is a model that predicts the coordinates of malignant pulmonary nodules and demarcates the associated areas using CT scans, for identifying malignant Lung Nodules and estimating their malignancy scores, the final model had a sensitivity of 86 percent.

Banerjee et al. [38] suggested a paradigm for tumor classification, with ANN, Random forests, and SVM as machine learning algorithms. Artificial neural networks are more accurate in both area and texture dependent features. As the precision is compared to the proposed model, it can be shown that accuracy has improved while recall has decreased. MATLAB R2017a was used for digital image analysis, and a Jupyter notebook was used for machine learning classification. Random Forest 79 percent, SVM 86 percent, and ANN 92 percent were the accuracy for regionbased features, while Random Forest 70 percent, SVM 80 percent, and ANN 96 percent were the accuracy for texturebased features.

A technique k-Nearest-Neighbors was developed by Maleki et al [39], for which a genetic algorithm was used to efficiently pick features, to reduce the dimensions of the dataset and to improve the speed of the classifier. The experimental approach is used to determine the best value for $\mathrm{k}$ to increase the precision of the proposed algorithm. Use of the proposed solution to the database for lung cancer shows $100 \%$ accuracy.
Reddy et al [40] propose a model that is successful in detecting the phases of lung cancer using machine learning algorithms. The model combines K-NN, Decision Trees, and Neural Networks structures with the bagging ensemble approach to improve overall prediction accuracy. As opposed to individual algorithms, the proposed model's estimated outcomes are more accurate. The versions with and without bagging are compared to draw conclusions. The bootstrap aggregating methodology improves the individual models' performance, with accuracy scores of 97\% (Decision Tree), $94 \%$, and $96 \%$ (K-NN) respectively (Neural Networks). The integrated model has a score of 0.98 for accuracy. The precision of the integrated model is increased by 3.33 percent.

Günaydin et al [41] proposed machine learning methods for detecting lung cancer nodules that used Principal Component Analysis, K-NN, SVM, Nave Bayes, Decision Trees, and ANN to detect anomaly. Then, both approaches were compared both after and without preprocessing. The experimental findings indicate that Artificial Neural Networks produce the best results with 82,43 percent accuracy after image processing, while Decision Tree produces the best results with 93,24 percent accuracy without image processing. Standard Digital Image Database, Japanese Society of Radiological Technology (JSRT) CT was used as the dataset (computed tomography).

Early identification of lung nodes from low dose computed tomography (LDCT) images was suggested by Elnakib et al [42]. Initially, the proposed device processes the raw data in order to increase the comparison between low-dose videos. The compact profound learning capabilities of various architectures, including Alex, VGG16 and VGG19 networks are then explored. A genetic algorithm (GA) is trained to identify the most important early detection features for optimizing the derived collection of features. In order to reliably diagnose lung nodules, various forms of classifiers are then checked. The method is validated using the I-ELCAP International Early Lung Cancer Action Project (ELCAP) in 320 photographs from 50 separate topics. With VGG19 and SVM classification, the system suggested achieves the highest 96.25 percent detection precision, 97.5 percent sensitivity and 95 percent specificity.

\section{MATERIAL AND METHODS}

\section{A. Dataset}

The data used in this work is a lung cancer dataset that was first released in and later made available in the UCI machine learning repository under the name "Lung Cancer Data set". This dataset was used to show the capability of the optimum discriminant plane in ill-posed situations. This dataset contains data on the pathological forms of lung cancer. It contains 32 observations on three forms of lung cancer using 56 elements[43].

\section{B. Classification Models}

To compare the output of the classifiers, three classification methods are used. The smallest number of features was used to attain higher efficiency. The classifier models are defined briefly. 


\section{Support Vector Machine}

Support Vector Machine is a supervised learning algorithm that uses the Classification method to analyze data and predicate patterns. The texture is divided into two categories or classes by the SVM classifier: regular and abnormal pictures [44]. It is used to effectively map the nodule. SVM is a margin classifier (hyperplane) that separates the two classes, which is why it is often referred to as a non-probabilistic binary classifier. The Support Vector is described as the training data point that is nearest to the classifier, and the Support Vector Machine is the maximum classifier. The gap between the cancer nodules and the hyperplane is as wide as possible [45][46].

\section{K-Nearest Neighbor Classifier}

The KNN algorithm is a supervised classification method. It's a simple algorithm that looks for the nearest fit. The database is compared to the comparison set. The test sample's mark is determined by the closest match of the $\mathrm{k}$ nearest neighbors. To calculate the distances between research samples and database samples, various distances such as Euclidean, cosine, similarity, and city block are used[47].

\section{Convolutional Neural Network}

$\mathrm{CNN}$ as a supervised deep learning tool, $\mathrm{CNN}$ is an excellent choice. This algorithm is suitable for multi-class classification and binary classification (for example, predicting whether or not a diagnostic picture contains a malignant tumor) [48][49]. CNNs are often used to solve a wide range of pattern and image recognition issues. This deep learning approach is effective and appropriate for visual data because of three key characteristics. To begin with, local receptive fields are perfectly matched to the image data specificity of being correlated geographically but uncorrelated globally. Second, since the convolution is applied to the entire image, mutual weights allow for significant parameter reduction without affecting image processing. Finally, a grid-structured image allows for data pooling operations that reduce data complexity without sacrificing valuable information [50][51].

\section{Proposed Model}

The paper suggests a model to predict and classify the lung cancer classes. The proposed model starts with data preprocessing, feature selection, classification and evaluating), figure (2) shows the block diagram for the proposed work.

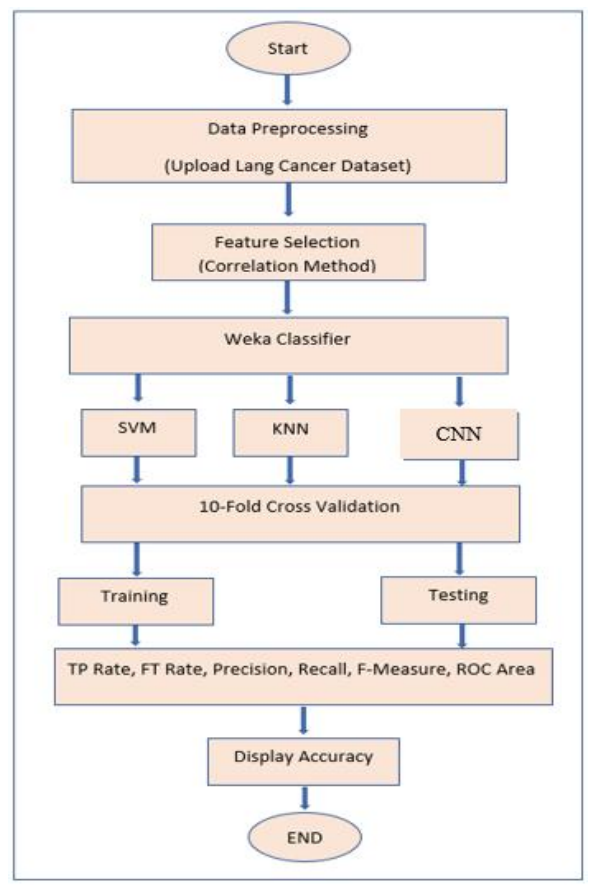

Fig. 2. Block Diagram for Proposed method

To define the lung cancer dataset in this article, Weka classifiers were used. WEKA was established by a team of researchers from New Zealand's University of Waikato [52]. It is a java-based open-source platform that can perform data mining and machine learning algorithms, such as data preprocessing, sorting, clustering, and association rule extraction, among other things. WEKA is a popular choice among analysts because of its ease of use and open-source nature [53].

In this paper for the feature selection Correlation Attribute (CA) method were used. CA is a feature subset selection algorithm [1]. It evaluates the attribute by calculating the correlation (Pearson's product moment correlation) between it and the class [4]. The main objective of CA is to obtain a highly relevant subset of features that are uncorrelated to each other. In this way, the dimensionality of datasets can be drastically reduced and the performance of learning algorithms can be improved [2]. Ranker search method used with CA. Based on the Correlation values, features are ranked and those features that are most suitable to be applied in the machine learning algorithm, are filtered [3].

\section{PERFORMANCE EVALUATION MATRICES}

\section{A. Confusion Matrix}

The Confusion Matrix is a deep learning visual assessment method. The prediction class results are represented in the columns of a Confusion Matrix, whereas the real class results are represented in the rows [54]. This matrix includes all the raw data regarding a classification model's assumptions on a specified data collection. To determine how accurate a model is. It's a square matrix with the rows representing the instances' real class and the columns representing their expected class. The confusion matrix is a $2 \times 2$ matrix that reports the number of true positives ( $\mathrm{T} \mathrm{P}$ ), true negatives ( $\mathrm{T} \mathrm{N}$ ), false positives (FP), 
and false negatives ( $\mathrm{F} \mathrm{N}$ ) when dealing with a binary

$$
\begin{array}{ll}
\text { T P } & \text { F N } \\
\text { FP } & \text { T N }
\end{array}
$$

Precision, recall, and F-measure, which are commonly utilized in the text mining and machine learning communities, were used to evaluate the algorithms. True positive (TP - objects correctly labeled as belonging to the class), false positive (FP - items falsely labeled as belonging to a certain class), false negative (FN - items incorrectly labeled as not belonging to a certain class), and true negative ( $\mathrm{TN}$ - items incorrectly labeled as not belonging to a certain class) are the four types of classified items (TN items correctly labelled as not belonging to a certain class). Recall is determined using the following formula given the amount of true positives and false negatives[55][56]:

$$
\text { Recall }=\frac{T P}{T P+F N}
$$

The recall is also known as "sensitivity" or the "absolute positive rate." Precision (also known as "positive predictive rate") is measured using the amount of true positive and false positive graded objects as follows:

$$
\text { Precision }=\frac{T P}{T P+F P}
$$

The measure that combines precision and recall is known as F-measure, given as:

$$
F=\frac{(1+\beta 2) \times \text { Recall } \times \text { Precision }}{\beta 2 \times(\text { Precision }+ \text { Recall })}
$$

where $\beta$ denotes the precision's relative value. A value of $\beta$ $=1$ (which is often used) means that recall and accuracy are of equal importance. A lower value implies that accuracy is more important, whereas a higher value indicates that recall is more important.

\section{B. ROC curve}

The region under the ROC curve, or literally AUC, summarizes the relationship between a binary classifier's true and false positive rate for various judgment thresholds. Several authors have shown that (AUC) is superior to absolute accuracy for classifier assessment, rendering it one of the most common metrics for static imbalanced data. To measure AUC, however, one must sort a specified dataset

and iterate through each example [57]. This ensures that AUC cannot be computed directly on vast data streams since it will necessitate scanning the whole stream after each example. As a result, the usage of AUC for data sources has been restricted to estimations on periodic holdout sets or whole streams, rendering it inherently skewed or computationally infeasible for realistic implementations [58].

\section{PERFORMANCE EVALUATION AND RESULTS}

The confusion matrix was used to evaluate the accuracy of each classifier. The experimental results show that using five attributes from an SVM classifier produces the best classification mission.

prediction ratio of 95.56 percent and, $\mathrm{CNN}$ accuracy ratio is 92.11 percent. While $\mathrm{KNN}$ has the lowest estimation percentage which is 88.40 percent.as shown in Table 2, 3, and 4. Table (2) shows and analysis the results for using SVM algorithm, Table (3) shows and analysis the results for using CNN algorithm.

TABLE 1. USING SVM CLASSIFIER

\begin{tabular}{|c|c|c|c|c|c|c|}
\hline \multirow{2}{*}{ Class } & \multicolumn{7}{|c|}{ using SVM classifier } \\
\cline { 2 - 7 } & $\begin{array}{c}\text { TP } \\
\text { Rate }\end{array}$ & $\begin{array}{c}\text { FP } \\
\text { Rate }\end{array}$ & Precision & Recall & $\begin{array}{c}\text { F- } \\
\text { Measure }\end{array}$ & $\begin{array}{c}\text { ROC } \\
\text { Area }\end{array}$ \\
\hline 1 & 0.986 & 0.109 & 0.951 & 0.986 & 0.968 & 0.946 \\
\hline 2 & 0.882 & 0.005 & 0.938 & 0.882 & 0.909 & 0.984 \\
\hline 3 & 0.667 & 0.000 & 1.000 & 0.667 & 0.800 & 0.968 \\
\hline 4 & 0.857 & 0.005 & 0.947 & 0.857 & 0.900 & 0.944 \\
\hline 5 & 1.000 & 0.000 & 1.000 & 1.000 & 1.000 & 1.000 \\
\hline Avg & 0.956 & 0.076 & 0.956 & 0.956 & 0.954 & 0.955 \\
\hline
\end{tabular}

TABLE 2. USING K-NEAREST NEIGHBOR CLASSIFIER

\begin{tabular}{|c|c|c|c|c|c|c|}
\hline \multirow{3}{*}{ Class } & \multicolumn{7}{|c|}{ using K-Nearest Neighbor Classifier } \\
\cline { 2 - 7 } & $\begin{array}{c}\text { TP } \\
\text { Rate }\end{array}$ & $\begin{array}{c}\text { FP } \\
\text { Rate }\end{array}$ & Precision & Recall & $\begin{array}{c}\text { F- } \\
\text { Measure }\end{array}$ & $\begin{array}{c}\text { ROC } \\
\text { Area }\end{array}$ \\
\hline 1 & 0.964 & 0.234 & 0.899 & 0.964 & 0.931 & 0.878 \\
\hline 2 & 0.706 & 0.016 & 0.800 & 0.706 & 0.750 & 0.892 \\
\hline 3 & 0.333 & 0.000 & 1.000 & 0.333 & 0.500 & 0.815 \\
\hline 4 & 0.762 & 0.011 & 0.889 & 0.762 & 0.821 & 0.887 \\
\hline 5 & 0.900 & 0.005 & 0.947 & 0.900 & 0.923 & 0.959 \\
\hline Avg & 0.897 & 0.164 & 0.898 & 0.897 & 0.891 & 0.881 \\
\hline
\end{tabular}

TABLE 3. USING CONVOLUTIONAL NEURAL NETWORK

\begin{tabular}{|c|c|c|c|c|c|c|}
\hline \multirow{3}{*}{ Class } & \multicolumn{6}{|c|}{ USING CONVOLUTIONAL NEURAL NETWORK } \\
\cline { 2 - 7 } & $\begin{array}{c}\text { TP } \\
\text { Rate }\end{array}$ & $\begin{array}{c}\text { FP } \\
\text { Rate }\end{array}$ & Precision & Recall & $\begin{array}{c}\text { F- } \\
\text { Measure }\end{array}$ & $\begin{array}{c}\text { ROC } \\
\text { Area }\end{array}$ \\
\hline 1 & 0.906 & 0.031 & 0.984 & 0.906 & 0.944 & 0.978 \\
\hline 2 & 0.882 & 0.027 & 0.750 & 0.882 & 0.811 & 0.987 \\
\hline 3 & 1.000 & 0.020 & 0.600 & 1.000 & 0.750 & 0.994 \\
\hline 4 & 0.952 & 0.016 & 0.870 & 0.952 & 0.909 & 0.988 \\
\hline 5 & 1.000 & 0.011 & 0.909 & 1.000 & 0.952 & 0.997 \\
\hline Avg & 0.921 & 0.027 & 0.934 & 0.921 & 0.924 & 0.982 \\
\hline
\end{tabular}

Table (4) show the comparison between the three classifiers depending on the time taken to build the model and the accuracy of the classifier.

TABLE 4. COMPARISON OF RESULT BY TIME AND ACCURACY

\begin{tabular}{|l|l|l|}
\hline \multirow{2}{*}{ CLASSIFIER } & \multicolumn{2}{|l|}{ COMPARISON OF RESULTS } \\
\cline { 2 - 3 } & $\begin{array}{l}\text { TIME TAKEN TO } \\
\text { BUILD MODEL }\end{array}$ & ACCURACY \\
\hline SVM & 1.77 SEC. & 95.56 \\
\hline KNN & 0.01 SEC. & 89.65 \\
\hline CNN & 3.79 SEC. & 92.11 \\
\hline
\end{tabular}

Figure (3) shows that CNN algorithm takes the longest time to build its model while $\mathrm{KNN}$ algorithm had the shortest time 


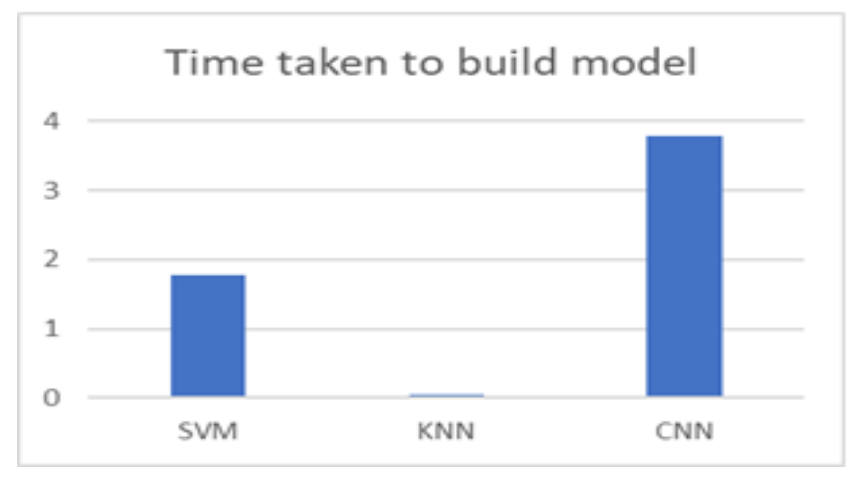

Fig. 3. Time analysis

FIGURE (4) SHOWS THAT SVM ALGORITHM HAD THE HIGHER ACCURACY. WHILE KNN'S ACCURACY HAS THE MINIMUM VALUE.

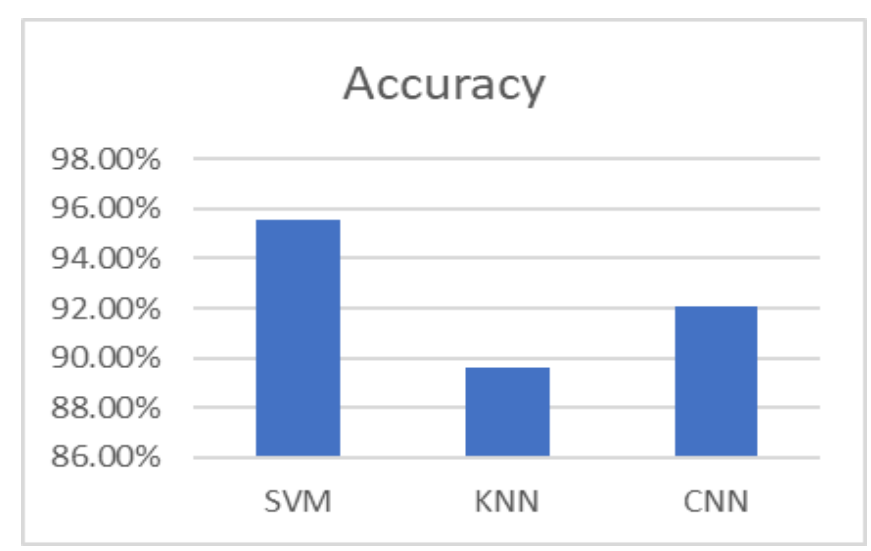

Fig. 4. ACCURACY ANALYSIS

\section{COMPARATIVE STUdiEs}

As shown in the table (5), the researchers used several different methods, different dataset and different ways of feature selection/feature extraction. in comparison with related work, we obtain a good result in this work with dataset and methods that we used. However, researcher in [13] obtained 94\% CT scan images dataset and SURF (Speeded Up Robust Features) for feature selection.in addition the researchers in [14],[15] they used the same dataset, the researchers in [14] obtained $90.9 \%$ with used Delta Radiomics method for feature extraction. But researchers in [15] could obtained better accuracy by using GLCM function for feature extraction and (MLP 98\%, SVM $70.45 \%$, \& KNN 99.2\%) classifier.by using (UCI) dataset the researchers in [6] could gain a good result $90 \%$ and used several classifiers.

Each of researchers in [16][17] they used same dataset (LIDC-IDRI) the researchers in[16] could obtained $86 \%$ sensitivity while, researchers in [17] depending on 2 features and applied different classifiers they obtained a good result (Random Forest 70\%, SVM 80\% and, ANN $96 \%$ ).also the researchers in [18],[19] by using the same dataset and number of feature selection which is(23), but the researchers in[18] obtained higher accuracy $100 \%$ by using KNN classifier and genetic algorithm for feature selection. while the researchers in [19] obtained (Decision Tree 97\%, K-NN 94\% and, Neural Networks 96\%). researchers in [20] used (JSRT) dataset and several classifiers to gain experimental results (ANN 82,43\% and, Decision Tree 93,24\%). finally, the researchers in [21] used (LDCT) dataset and smart genetic algorithm with applying (SVM) to obtained $96.25 \%$ accuracy. 


\begin{tabular}{|c|c|c|c|c|c|c|}
\hline \multirow{2}{*}{ Ref } & \multicolumn{6}{|c|}{ Comparison of Related Work } \\
\hline & Dataset & Feature No. & Feature Selection & Feature Extraction & Classifier & Result \\
\hline Roy et al[34] & CT scan images & - & $\begin{array}{l}\text { SURF (Speeded } \\
\text { Up Robust } \\
\text { Features) }\end{array}$ & 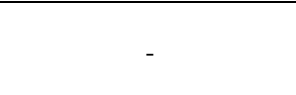 & $\begin{array}{l}\text { random forest algorithm and } \\
\text { SVM classification }\end{array}$ & $94.5 \%$ \\
\hline Faisal et al [12] & UCI & - & - & - & $\begin{array}{c}\text { (SVM), C4.5, Multi-Layer } \\
\text { Perceptron, Decision tree, } \\
\text { Naïve Bayes, and Neural } \\
\text { Network }\end{array}$ & $90 \%$ \\
\hline Baskar et al [35] & CT images & - & - & $\begin{array}{l}\text { features extracted by } \\
\text { Delta Radiomics }\end{array}$ & SVM & $90.9 \%$ \\
\hline Boban et al[36] & (CT) images & 8 & - & $\begin{array}{l}\text { features extracted using } \\
\text { GLCM function }\end{array}$ & $\begin{array}{c}\text { MLP, } \\
\text { SVM, } \\
\text { \& KNN }\end{array}$ & $\begin{array}{c}\text { MLP 98\% } \\
\text { SVM } 70.45 \% \\
\text { KNN } 99.2 \% \\
\end{array}$ \\
\hline $\begin{array}{l}\text { Sreekumar et } \\
\text { al[37] }\end{array}$ & LIDC-IDRI & - & - & $\begin{array}{l}\text { 3D CNN model based on } \\
\text { the C3D network } \\
\text { architecture }\end{array}$ & $\mathrm{CNN}$ & sensitivity $86 \%$ \\
\hline Banerjee et al[38] & LIDC-LDRI & 2 & - & & $\begin{array}{l}\text { Random Forest, } \\
\text { SVM and, } \\
\text { ANN }\end{array}$ & $\begin{array}{c}\text { Random Forest 70\% } \\
\text { SVM } 80 \% \\
\text { ANN } 96 \%\end{array}$ \\
\hline Maleki et al [39] & $\begin{array}{l}\text { Data World } \\
\text { source }\end{array}$ & 23 & $\begin{array}{l}\text { genetic algorithm } \\
\text { for feature } \\
\text { selection }\end{array}$ & - & KNN & $100 \%$ \\
\hline Reddy et at[40] & $\begin{array}{l}\text { Data World } \\
\text { source }\end{array}$ & 23 & - & - & $\begin{array}{c}\text { Decision Tree, } \\
\text { K-NN and, } \\
\text { Neural Networks }\end{array}$ & $\begin{array}{c}\text { Decision Tree 97\% } \\
\text { K-NN 94\% } \\
\text { (Neural Networks } \\
96 \% \\
\end{array}$ \\
\hline $\begin{array}{l}\text { Günaydin et } \\
\text { al[41] }\end{array}$ & JSRT & - & - & - & $\begin{array}{c}\text { K-NN, } \\
\text { SVM, } \\
\text { Naïve Bayes, } \\
\text { Decision Trees } \\
\&, \\
\text { Artificial Neural Networks }\end{array}$ & $\begin{array}{c}\text { The experimental } \\
\text { results } \\
\text { ANN } 82,43 \% \\
\text { Decision Tree } 93,24 \%\end{array}$ \\
\hline Elnakib et al[42] & LDCT images & - & $\begin{array}{l}\text { smart genetic } \\
\text { algorithm }\end{array}$ & - & SVM & $96.25 \%$ \\
\hline This work & UCI & - & $\begin{array}{l}\text { Correlation } \\
\text { Method }\end{array}$ & - & $\begin{array}{l}\text { SVM } \\
\text { KNN } \\
\text { CNN }\end{array}$ & $\begin{array}{l}\text { SVM 95.56 } \\
\text { KNN 88.40 } \\
\text { CNN 92.11 }\end{array}$ \\
\hline
\end{tabular}

\section{CONCLUSION}

Lung cancer is one of the most dangerous diseases and the most common cause of death, the severity of the disease lies in the difficulty of diagnosing it in the early stages. This paper tries to endeavor to investigate of three classifiers to find the best classifier could classify lung cancer in early stage. The informational indices included in this study were

\section{REFERENCES}

[1] P. Chaudhari, H. Agarwal, and V. Bhateja, "Data augmentation for cancer classification in oncogenomics: an improved KNN based approach,” Evol. Intell., pp. 1-10, 2019.

S. F. Khorshid and A. M. Abdulazeez, "BREAST CANCER DIAGNOSIS BASED ON K-NEAREST NEIGHBORS: A REVIEW," PalArch's J. Archaeol. Egypt/Egyptology, vol. 18, no. 4, pp. 1927-1951, 2021.

[3] F. Q. Kareem and A. M. Abdulazeez, "Ultrasound Medical Images Classification Based on Deep Learning Algorithms: A Review."

[4] D. Q. Zeebaree, A. M. Abdulazeez, D. A. Zebari, H. Haron, and H. N. A. Hamed, "Multi-Level Fusion in Ultrasound for Cancer Detection Based on Uniform LBP Features." derived from UCI databases for lung cancer patients. The focus of this paper is on using WEKA Tool to investigate the accuracy of classification algorithms. The results show that the Support Vector Machine (SVM) give the best accuracy $95.56 \%$, that can detect lung cancer in its early stages and save several lives and, K-Nearest Neighbor KNN It gave less accuracy $88.40 \%$.

[5] J. R. F. Junior, M. Koenigkam-Santos, F. E. G. Cipriano, A. T. Fabro, and P. M. de Azevedo-Marques, "Radiomics-based features for pattern recognition of lung cancer histopathology and metastases," Comput. Methods Programs Biomed., vol. 159, pp. 23-30, 2018.

[6] I. Ibrahim and A. Abdulazeez, "The Role of Machine Learning Algorithms for Diagnosing Diseases,” J. Appl. Sci. Technol. Trends, vol. 2, no. 01, pp. 10-19, 2021.

[7] P. Das, B. Das, and H. S. Dutta, "Prediction of Lungs Cancer Using Machine Learning," EasyChair, 2020.

[8] G. A. P. Singh and P. K. Gupta, "Performance analysis of various machine learning-based approaches for detection and classification of lung cancer in humans," Neural Comput. Appl., vol. 31 , no. 10 , pp. $6863-6877,2019$. 
[9] B. Charbuty and A. Abdulazeez, "Classification Based on Decision Tree Algorithm for Machine Learning," J. Appl. Sci. Technol. Trends, vol. 2, no. 01, pp. 20-28, 2021.

[10] H. A. Hussein and A. M. Abdulazeez, "COVID-19 PANDEMIC DATASETS BASED ON MACHINE LEARNING CLUSTERING ALGORITHMS: A REVIEW," PalArch's J. Archaeol. Egypt/Egyptology, vol. 18, no. 4, pp. 2672-2700, 2021.

[11] D. M. Abdullah and N. S. Ahmed, "A Review of most Recent Lung Cancer Detection Techniques using Machine Learning," Int. J. Sci. Bus., vol. 5, no. 3, pp. 159-173, 2021.

[12] M. I. Faisal, S. Bashir, Z. S. Khan, and F. H. Khan, “An evaluation of machine learning classifiers and ensembles for early stage prediction of lung cancer," in 2018 3rd International Conference on Emerging Trends in Engineering, Sciences and Technology (ICEEST), 2018, pp. 1-4.

[13] D. Q. Zeebaree, H. Haron, and A. M. Abdulazeez, "Gene selection and classification of microarray data using convolutional neural network," in 2018 International Conference on Advanced Science and Engineering (ICOASE), 2018, pp. 145150.

[14] D. Q. Zeebaree, H. Haron, A. M. Abdulazeez, and D. A. Zebari, "Trainable model based on new uniform LBP feature to identify the risk of the breast cancer," in 2019 International Conference on Advanced Science and Engineering (ICOASE), 2019, pp. 106-111.

[15] H. Tang, J. Zhao, and X. Yang, "Explore machine learning for analysis and prediction of lung cancer related risk factors," in Proceedings of the 2018 2nd International Conference on Computer Science and Artificial Intelligence, 2018, pp. 41-45.

[16] P. R. Radhika, R. A. S. Nair, and G. Veena, "A Comparative Study of Lung Cancer Detection using Machine Learning Algorithms," in 2019 IEEE International Conference on Electrical, Computer and Communication Technologies (ICECCT), 2019, pp. 1-4.

[17] A. I. Rahmani and M. Katouli, "Diagnosing Lung Cancer Using Grasshopper Optimization Algorithm and k-Nearest Neighbor Classification," J. homepage http//iieta. org/journals/rces, vol. 6, no. 4, pp. 69-75, 2019.

[18] Y. Nai et al., "Improving Lung Lesion Detection in Low Dose Positron Emission Tomography Images Using Machine Learning," in 2018 IEEE Nuclear Science Symposium and Medical Imaging Conference Proceedings (NSS/MIC), 2018, pp. $1-3$

[19] S. Senthil and B. Ayshwarya, "Lung cancer prediction using feed forward back propagation neural networks with optimal features," Int. J. Appl. Eng. Res., vol. 13, no. 1, pp. 318-325, 2018.

[20] [20]M. R. Mahmood, A. M. Abdulazeez, and Z. ORMAN, “A NEW HAND GESTURE RECOGNITION SYSTEM USING ARTIFICIAL NEURAL NETWORK."

[21] M. Somvanshi, P. Chavan, S. Tambade, and S. V. Shinde, "A review of machine learning techniques using decision tree and support vector machine," Proc. - 2nd Int. Conf. Comput. Commun. Control Autom. ICCUBEA 2016, 2017, doi: 10.1109/ICCUBEA.2016.7860040.

[22] D. M. Abdulqader, A. M. Abdulazeez, and D. Q. Zeebaree, "Machine Learning Supervised Algorithms of Gene Selection: A Review," Mach. Learn., vol. 62, no. 03, 2020.

[23] O. Ahmed and A. Brifcani, "Gene Expression Classification Based on Deep Learning," in 2019 4th Scientific International Conference Najaf (SICN), 2019, pp. 145-149.

[24] N. O. M. Salim and A. M. Abdulazeez, "Human Diseases Detection Based On Machine Learning Algorithms: A Review," Int. J. Sci. Bus., vol. 5, no. 2, pp. 102-113, 2021.

[25] N. M. Abdulkareem and A. M. Abdulazeez, "Machine Learning Classification Based on Radom Forest Algorithm: A Review,” Int. J. Sci. Bus., vol. 5, no. 2, pp. 128-142, 2021.

[26] R. Sathishkumar, K. Kalaiarasan, A. Prabhakaran, and M. Aravind, "Detection of Lung Cancer using SVM Classifier and KNN Algorithm," in 2019 IEEE International Conference on System, Computation, Automation and Networking (ICSCAN), 2019, pp. 1-7.

[27] S. Uddin, A. Khan, M. E. Hossain, and M. A. Moni, "Comparing different supervised machine learning algorithms for disease prediction,” BMC Med. Inform. Decis. Mak., vol. 19, no. 1 , pp. 1-16, 2019.

[28] N. Najat and A. M. Abdulazeez, "Gene clustering with partition around mediods algorithm based on weighted and normalized Mahalanobis distance," in 2017 International Conference on Intelligent Informatics and Biomedical Sciences (ICIIBMS), 2017, pp. 140-145.

[29] S. Hussein, P. Kandel, C. W. Bolan, M. B. Wallace, and U. Bagci, "Lung and pancreatic tumor characterization in the deep learning era: novel supervised and unsupervised learning approaches,” IEEE Trans. Med. Imaging, vol. 38, no. 8, pp. 1777-1787, 2019

[30] B. M. S. Hasan and A. M. Abdulazeez, "A Review of Principal Component Analysis Algorithm for Dimensionality Reduction," J. Soft Comput. Data Min., vol. 2, no. 1, pp. 20-30, 2021.

[31] D. M. Sulaiman, A. M. Abdulazeez, H. Haron, and S. S. Sadiq, "Unsupervised Learning Approach-Based New Optimization K-Means Clustering for Finger Vein Image Localization," in 2019 International Conference on Advanced Science and Engineering (ICOASE), 2019, pp. 82-87.

[32] H. U. Dike, Y. Zhou, K. K. Deveerasetty, and Q. Wu, "Unsupervised learning based on artificial neural network: A review," in 2018 IEEE International Conference on Cyborg and Bionic Systems (CBS), 2018, pp. 322-327.

[33] H. R. Abdulqadir and A. M. Abdulazeez, "Reinforcement Learning and Modeling Techniques: A Review," Int. J. Sci. Bus. vol. 5, no. 3, pp. 174-189, 2021.

[34] K. Roy et al., "A Comparative study of Lung Cancer detection using supervised neural network," in 2019 Internationa Conference on Opto-Electronics and Applied Optics (Optronix), 2019, pp. 1-5.

[35] S. Baskar, P. M. Shakeel, K. P. Sridhar, and R. Kanimozhi, "Classification System for Lung Cancer Nodule Using Machine Learning Technique and CT Images," in 2019 International Conference on Communication and Electronics Systems (ICCES), 2019, pp. 1957-1962.

[36] B. M. Boban and R. K. Megalingam, "Lung Diseases Classification based on Machine Learning Algorithms and Performance Evaluation," in 2020 International Conference on Communication and Signal Processing (ICCSP), 2020, pp. 315320 .

[37] A. Sreekumar, K. R. Nair, S. Sudheer, H. G. Nayar, and J. J. Nair, "Malignant Lung Nodule Detection using Deep Learning," in 2020 International Conference on Communication and Signal Processing (ICCSP), 2020, pp. 209-212.

[38] N. Banerjee and S. Das, "Prediction Lung Cancer-In Machine Learning Perspective," in 2020 International Conference on Computer Science, Engineering and Applications (ICCSEA), 2020, pp. 1-5.

[39] N. Maleki, Y. Zeinali, and S. T. A. Niaki, "A k-NN method for lung cancer prognosis with the use of a genetic algorithm for feature selection," Expert Syst. Appl., vol. 164, p. 113981, 2021.

[40] D. Reddy, E. N. H. Kumar, D. Reddy, and P. Monika, "Integrated Machine Learning Model for Prediction of Lung Cancer Stages from Textual data using Ensemble Method," in 2019 1st International Conference on Advances in Information Technology (ICAIT), 2019, pp. 353-357.

[41] Ö. Günaydin, M. Günay, and Ö. Şengel, "Comparison of lung cancer detection algorithms," in 2019 Scientific Meeting on Electrical-Electronics \& Biomedical Engineering and Computer Science (EBBT), 2019, pp. 1-4.

[42] A. Elnakib, H. M. Amer, and F. E. Z. Abou-Chadi, "Early Lung Cancer Detection Using Deep Learning Optimization," 2020.

[43] S. M. Salaken, A. Khosravi, A. Khatami, S. Nahavandi, and M. A. Hosen, "Lung cancer classification using deep learned features on low population dataset," in 2017 IEEE 30th Canadian Conference on Electrical and Computer Engineering (CCECE), 2017, pp. 1-5.

[44] A. Asuntha and A. Srinivasan, "Deep learning for lung Cancer detection and classification," Multimed. Tools Appl., vol. 79, no. 11, pp. 7731-7762, 2020.

[45] W. Rahane, H. Dalvi, Y. Magar, A. Kalane, and S. Jondhale, "Lung cancer detection using image processing and machine learning healthcare," in 2018 International Conference on Current Trends towards Converging Technologies (ICCTCT), 2018, pp. $1-5$.

[46] H. S. Yahia and A. M. Abdulazeez, "Medical Text Classification Based on Convolutional Neural Network: A Review,” Int. J. Sci. Bus., vol. 5, no. 3, pp. 27-41, 2021. 

Perceptron Based Lung Tumor Classification," in 2018 Second International Conference on Electronics, Communication and Aerospace Technology (ICECA), 2018, pp. 499-502.

[48] S. S. Raoof, M. A. Jabbar, and S. A. Fathima, "Lung Cancer Prediction using Machine Learning: A Comprehensive Approach," in 2020 2nd International Conference on Innovative Mechanisms for Industry Applications (ICIMIA), 2020, pp. 108115.

[49] J. Saeed and A. M. Abdulazeez, "Facial Beauty Prediction and Analysis Based on Deep Convolutional Neural Network: A Review," J. Soft Comput. Data Min., vol. 2, no. 1, pp. 1-12, 2021.

[50] Y. Lei, B. Yang, X. Jiang, F. Jia, N. Li, and A. K. Nandi, "Applications of machine learning to machine fault diagnosis: A review and roadmap,” Mech. Syst. Signal Process., vol. 138, p. $106587,2020$.

[51] N. Omar, A. M. Abdulazeez, A. Sengur, and S. G. S. Al-Ali, "Fused faster RCNNs for efficient detection of the license plates," Indones. J. Electr. Eng. Comput. Sci., vol. 19, no. 2, pp. 974-982, 2020.

[52] Z. Zainudin, S. M. Shamsuddin, and S. Hasan, "Deep Learning for Image Processing in WEKA Environment," Int. J. Adv. Soft Compu. Appl, vol. 11, no. 1, 2019.

[53] V. Mhetre and M. Nagar, "Classification based data mining algorithms to predict slow, average and fast learners in educational system using WEKA," in 2017 International Conference on Computing Methodologies and Communication (ICCMC), 2017, pp. 475-479.

[54] Al Janabi, K. B., \& Kadhim, R. (2018). Data reduction techniques: a comparative study for attribute selection methods.
International Journal of Advanced Computer Science and Technology, 8(1), 1-13.

[55] Sugianela, Y., \& Ahmad, T. (2020, February). Pearson Correlation Attribute Evaluation-based Feature Selection for Intrusion Detection System. In 2020 International Conference on Smart Technology and Applications (ICoSTA) (pp. 1-5). IEEE.

[56] Demisse, G. B., Tadesse, T., \& Bayissa, Y. (2017). Data mining attribute selection approach for drought modeling: A case study for Greater Horn of Africa. arXiv preprint arXiv:1708.05072.

[57] Kumar, S., \& Chong, I. (2018). Correlation analysis to identify the effective data in machine learning: Prediction of depressive disorder and emotion states. International journal of environmental research and public health, 15(12), 2907.

[58] O. Caelen, "A Bayesian interpretation of the confusion matrix," Ann. Math. Artif. Intell., vol. 81, no. 3, pp. 429-450, 2017

[59] N. Milosevic, A. Dehghantanha, and K.-K. R. Choo, "Machine learning aided Android malware classification," Comput. Electr. Eng., vol. 61, pp. 266-274, 2017.

[60] J. Xu, Y. Zhang, and D. Miao, "Three-way confusion matrix for classification: A measure driven view," Inf. Sci. (Ny)., vol. 507, pp. 772-794, 2020

[61] Z. Yang, T. Zhang, J. Lu, D. Zhang, and D. Kalui, "Optimizing area under the ROC curve via extreme learning machines," Knowledge-Based Syst., vol. 130, pp. 74-89, 2017.

[62] D. Brzezinski and J. Stefanowski, "Prequential AUC: properties of the area under the ROC curve for data streams with concept drift,” Knowl. Inf. Syst., vol. 52, no. 2, pp. 531-562, 2017. 